ATLAS Internal Note

INDET-NO-134

25 June 1996

\title{
Radiation Damage due to NIEL in GaAs Particle Detectors.
}

\author{
A Chilingarov ${ }^{1}$, J S Meyer and T Sloan \\ School of Physics \& Chemistry \\ Lancaster University \\ Lancaster LA1 4YB, UK
}

Presented at 4th International Workshop on Gallium Arsenide and Related Compounds,

Aberfoyle (1996)

\begin{abstract}
The Non Ionizing Energy Loss (NIEL) for fast neutrons, protons and pions in GaAs has been deduced from published calculations. The values are then used to search for a correlation between the observed reduction of charge collection efficiency (CCE) in GaAs particle detectors with the radiation dose from NIEL. A correlation is demonstrated to be present for detectors made from a wide range of material. The implications for the performance of GaAs detectors at the Large Hadron Collider (LHC) are discussed.
\end{abstract}

\section{Introduction}

It is well known that the observed radiation damage to electronic components made from Gallium Arsenide is proportional to the Non-Ionising Energy Loss (NIEL) [1]. It is possible that this proportionality is also valid for GaAs particle detectors which have been subjected to large doses of radiation from hadronic particles. Such damage has been observed in detectors irradiated by $\mathrm{MeV}$ neutrons [2] and more recently by fast protons and pions $[3,4,5]$. In contrast GaAs detectors are able to withstand very large doses (megarads) of gamma radiation with almost no impairment of their performance [2]. Such gamma radiation produces little NIEL [6]. In this paper we investigate the

\footnotetext{
${ }^{1}$ On leave from the Budker Institute for Nuclear Physics, Novosibirsk, Russia
} 
correlation between the NIEL from strongly interacting particles and the performance of GaAs detectors as measured by their charge collection efficiency (CCE).

A charged particle passing through matter loses energy both by interactions with atomic electrons and by collisions with nuclei in the medium. The latter is the nonionizing energy loss (NIEL). High energy particles with velocity $v$ much greater than the velocity of the electrons in the atom lose the majority of their energy by electronic interactions, whilst for low energy particles the loss is principally due to nuclear collisions (NIEL). High energy particles do lose some energy to NIEL due to the low energy products of nuclear interactions. Such energy loss is roughly 3 orders of magnitude smaller than that due to ionization effects from interactions with atomic electrons. However, ionization effects are fully recoverable whilst a part of the NIEL produces permanent damage due to atoms being either destroyed or knocked out of the lattice.

The NIEL for a particle passing through matter must be calculated. The values of the stopping power due to NIEL are given by

$$
\frac{d E}{d x}{ }_{N}=\sum_{Z, A} \int E_{r} \frac{N}{A^{\prime}} \frac{d \sigma}{d E_{r}} L\left(E_{r}\right) d E_{r}
$$

where $N$ and $A^{\prime}$ are Avogadro's number and the atomic weight of the medium, $\frac{d \sigma}{d E_{r}}$ is the differential cross section to produce a recoil fragment with energy $E_{r}$ of atomic weight (number) $\mathrm{A}(\mathrm{Z})$ and the function $L\left(E_{r}\right)$ is the fraction of this energy which appears as NIEL at a particular energy. This function is usually calculated according to the prescription of Lindhard et al [7]. The calculations of the NIEL stopping power are difficult because information on the nuclear break up following a collision has to be modelled to

obtain $\frac{d \sigma}{d E_{r}}$. Such modelling needs a detailed understanding of the nuclear physics of the process and hence incurs some uncertainty.

\section{The Values of the NIEL stopping power}

Extensive calculations have been performed for Silicon $[6,8,9,10]$ since most particle detectors have previously been made of such material. Calculations [9,10] exist for neutrons in $\mathrm{GaAs}$ and these are shown in fig 1 together with the results for Si. The detectors discussed here were irradiated at the ISIS irradiation facility [11]. To deduce the NIEL from this source of neutrons the data in fig 1 were integrated over the measured spectrum from the source [11]. The different computations are given in table 1. Comparing them 
shows that the accuracy of the NIEL stopping power for neutrons is about $10 \%$.

Table 1 Calculated NIEL stopping power for neutrons

Reference

$$
\begin{array}{cc}
\frac{d E}{d x}{ }_{N} \text { ISIS neutrons } & \frac{d E}{d x} N_{N} 1 \mathrm{MeV} \text { neutrons } \\
\left(\mathrm{keV}\left(\mathrm{gm} \mathrm{cm}^{-2}\right)^{-1}\right) & \left(\mathrm{keV}\left(\mathrm{gm} \mathrm{cm}^{-2}\right)^{-1}\right)
\end{array}
$$

\section{Silicon}

$\begin{array}{lccc}\text { Van Ginneken } & {[6]} & 1.95 & 1.57 \\ \text { Luera et al } & {[9]} & 2.17 & 1.98 \\ \text { Ougouag et al } & {[10]} & 1.96 & 1.62\end{array}$

\section{GaAs}

$\begin{array}{lccc}\text { Luera et al } & {[9]} & 0.81 & 0.513 \\ \text { Ougouag et al } & {[10]} & 0.86 & 0.583\end{array}$

For energetic charged particles in GaAs calculations only exist for protons up to a kinetic energy of $1 \mathrm{GeV}$ [1]. To extend the range of these computations we assume that the values in GaAs will follow the same variation with energy as those in Silicon so that

$$
\frac{d E}{d x}{ }_{N}(\text { Energy E in GaAs })=\frac{\frac{d E}{d x}{ }_{N}(\mathrm{GaAs} 1 \mathrm{GeV}) \times \frac{d E}{d x}{ }_{N}(\text { Energy E in Si })}{\frac{d E}{d x}{ }_{N}(\mathrm{Si} 1 \mathrm{GeV})}
$$

To obtain $\frac{d E}{d x}{ }_{N}$ for pions in GaAs we assume that the ratio of the $\frac{d E}{d x}{ }_{N}$ for protons to that for pions in GaAs will be the same as that in $\mathrm{Si}$. We then use the calculated $\mathrm{Si}$ ratios from [8] and $\frac{d E}{d x}{ }_{N}$ for protons in GaAs obtained from equation 2 to calculate $\frac{d E}{d x}{ }_{N}$ for pions in GaAs.

Fig 2 shows the results of this procedure for pions and protons together with the values for Si given in [8]. The accuracy of these curves will be poorer than for low energy neutrons which cause simpler final states of the recoil nucleus following inelastic scattering. For protons in Si the curve is based on experimental data which at energies above $1 \mathrm{GeV}$ gives somewhat lower damage factors than those predicted in [6]. This deficit is consistent with the findings of the recent survey in [18]. The accuracy of the curve for protons in $\mathrm{Si}$ is expected to be about $30 \%$. For pions in Si the accuracy will be somewhat worse due to the above mentioned uncertainties. Fig 3 shows the pion curve from fig 2 together with the recent computations for pions in Si reported in [12]. Differences of $50 \%$ can be seen between the two. Hence the accuracy of the pion computations in $\mathrm{Si}$ is likely to be at least of this order. For GaAs the computations depend on one source [1]. The accuracy 
is likely to be similar to that in Si but with the added extrapolations and assumptions made here uncertainties of order a factor of 2 are not unlikely.

From the curves in figure 2 the NIEL stopping powers for the irradiation types described in $[3,4,5]$ can be deduced and these are shown in table 2. The values for silicon are also included for comparison.

\section{Table 2}

\begin{tabular}{|c|c|c|}
\hline $\begin{array}{l}\text { Irradiation } \\
\text { type. }\end{array}$ & 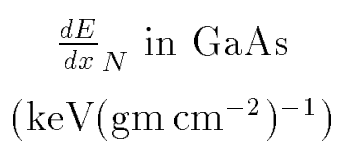 & $\begin{array}{c}\frac{d E}{d x}{ }_{N} \text { in } \mathrm{Si} \\
\left(\mathrm{keV}\left(\mathrm{gm} \mathrm{cm}^{-2}\right)^{-1}\right)\end{array}$ \\
\hline ISIS neutrons. & 0.9 & 2.0 \\
\hline $24 \mathrm{GeV} / \mathrm{c}$ protons. & 2.9 & 1.0 \\
\hline $300 \mathrm{MeV} / \mathrm{c}$ pions. & 3.6 & 1.9 \\
\hline
\end{tabular}

\section{Comparison with Measurements of the CCE}

The measurements of the charge collection efficiency (CCE) described in [3] are shown as a function of particle fluence (particles $\mathrm{cm}^{-2}$ ) in fig 4 for irradiations by ISIS neutrons, $300 \mathrm{MeV} / \mathrm{c}$ pions and $24 \mathrm{GeV} / \mathrm{c}$ protons. It can be seen that the CCE seems to fall with fluence but at a different rate for pions, protons and neutrons.

The total NIEL deposited per unit mass of the detectors is the product of the fluence and the NIEL stopping power, $\frac{d E}{d x}{ }_{N}$. Fig 5 shows the CCE plotted against total NIEL using the stopping powers given in table 2. The data in fig 5 follow a smoother behaviour than those in fig 4 with the CCE falling at similar rates of irradiation for pions, protons and neutrons. This implies a correlation between the CCE and total NIEL dose.

To test statistically the significance of this correlation a procedure was adopted to deduce the experimental errors from the scatter of the data. The data for each irradiation type was fitted to a regression line and the slope obtained. The measurements of fluence and total NIEL for each detector were interpolated to a fixed CCE (chosen as $35 \%$, ie roughly at the centre of the range) using this slope. The standard deviation of these calculated values gave a measure of the error on each measurement for each irradiation type.

A $\chi^{2}$ test was then made to the hypotheses a) that the neutron, pion and proton irradiations fall at the same rate with fluence; b) that the three fall at the same rate with 
total NIEL. This was done by calculating the weighted means of the fluence and the total NIEL for $35 \%$ CCE. The $\chi^{2}$ of the three irradiation types to these means was derived and the confidence level of each hypothesis obtained. The results are shown in table 3.

\section{Results}

\section{Table 3}

$\begin{array}{lccccc}\begin{array}{l}\text { Irradiation:- } \\ \text { type }\end{array} & \mathrm{n} & \text { pion } & \text { proton } & \text { weighted mean } & \begin{array}{c}\chi^{2} \\ \text { to mean }\end{array} \\ \text { Mean fluence } & & & & & \\ \text { for } 35 \% \mathrm{CCE} & 2.30 \pm .27 & 0.50 \pm 0.08 & .85 \pm .13 & .70 \pm .066 & 43.4 / 2 \mathrm{dof} \\ \left(10^{14} \mathrm{~cm}^{-2}\right) & & & & & \\ & & & & & \\ \text { Mean total } & & & & & \\ \text { NIEL for } & 2.07 \pm 0.24 & 1.8 \pm 0.3 & 2.5 \pm 0.4 & 2.06 \pm 0.17 & \\ \left.35 \% \mathrm{CCE}^{-1}\right) & & & & & \end{array}$

The $\chi^{2}$ to the hypothesis that the irradiation types fall at the same rate with fluence of 43.4 for 2 degrees of freedom (dof) has a probability of less than $10^{-4}$. This proves statistically that this hypthesis is false. On the other hand that for the fall with total NIEL of 1.9 for 2 dof has a probability of $40 \%$ showing that the data are consistent with the same rate of fall of CCE for each type of irradiation. This confirms statistically the existence of a correlation between the CCE and the total NIEL dose.

Another way to interpret these data is to assume that the CCE is correlated absolutely to the total NIEL. The ratio of the fluences for $35 \% \mathrm{CCE}$ can then be used to measure the ratio of the NIEL stopping powers for the three irradiation types. This would give measured ratios $\mathrm{n}: \pi: \mathrm{p}$ of $1.0: 4.6 \pm 0.9: 2.7 \pm 0.5$ in good agreement with the calculated ratios of 1.0:4.0:3.2. Further confirmation of this effect comes from the measurements of the fluences to reduce the total signal from minimum ionizing particles (MIPs) to 8000 electrons in the Aachen detectors [4]. This was found to be $4.610^{14}, 1.210^{14}$ and $1.510^{14}$ 
for neutrons, pions and protons $\mathrm{cm}^{-2}$, respectively. Hence, again assuming that the CCE is correlated to the total NIEL, these data imply that the ratios of the NIEL stopping powers is 1.0:3.8:3.1 in good agreement with the calculated values.

Fig 6 shows the data displayed in fig 5 together with the average CCE of the detectors before irradiation. The data seem to show an initial fast fall with total NIEL dose followed by a slower rate of fall. The smooth curve shows a fit of the form

$$
C C E=38 e^{-2.5 T N}+46 e^{-0.17 T N}
$$

where TN is the total NIEL dose in keV per gram. This fit can be used to compare the data from the Glasgow detectors [3] with those from other measurements $[4,5,15]$.

Figs 7-10 show the measurements reported by the Aachen [4] and Freiburg [5] groups. The smooth curves in these figures are calculated from equation 3 , converting to fluences for figs 7-9 and to NIEL relative to $1 \mathrm{Mev}$ neutrons in fig 10 using the data for GaAs in table 2. In each case the normalization of the curve is unchanged from that for the Glasgow detectors which gave an average signal of 22.3 thousand electrons for MIPs passing through a $200 \mu \mathrm{m}$ thick detector, before irradiation. (The exception is the lower plot in fig 9 which is arbitrarily normalized to the data at zero fluence since this was a $600 \mu \mathrm{m}$ thick detector). There is reasonable agreement between the curves and all the data within the experimental uncertainties. Hence the correlation between the total NIEL and the CCE observed for the data from [3] gives a quantitatively reasonable agreement with the data from $[4,5]$. Furthermore, detectors with a $\pi-\nu$ structure which have been fabricated in Tomsk show the same effect. The deterioration in the signal after irradiation by $310^{14}$ neutrons per $\mathrm{cm}^{2}$ at ISIS was observed to be similar to that produced by $1.110^{14}$ $24 \mathrm{GeV} / \mathrm{c}$ protons $\mathrm{cm}^{-2}[15]$, in good agreement with the ratio expected from the NIEL stopping powers in table 2 and at a rate compatible with the curve in fig 6 .

The fact that a wide range of data from many different types of detectors can be well reproduced assuming that a correlation exists between the CCE and the total NIEL dose is strong evidence for the correlation. Possible exceptions to this general conclusion are the detectors made from the FCM-LC material shown in figs 7 and 8 which seem to deteriorate slightly less rapidly under irradiation than all the other detectors. In addition, the most recent $\pi-\nu$ detectors from Tomsk seem to show exceptional radiation hardness 
[17]. This is probably due to the very specific technology used in the production of these detectors.

\section{Discussion of the Results.}

It seems unlikely that the correlation between CCE and NIEL, observed in such a wide range of detectors made from many different sources of GaAs, is accidental. Furthermore, such a correlation can be explained as follows.

It is known that in Si the NIEL stopping powers for $1 \mathrm{MeV}$ neutrons and relativistic protons and pions have similar values. Their damage factors are also almost equal to each other [18]. However, GaAs particle detectors show good radiation hardness to $1 \mathrm{Mev}$ neutrons but their much stronger sensitivity to protons and pions has come as something of a surprise. We can understand this in terms of the Lindhard factors in the material and the interaction cross sections. It can be seen from fig 1 that the NIEL for 1 Mev neutrons in $\mathrm{Si}$ is larger than that in GaAs. This is due to the fact that the $1 \mathrm{Mev}$ neutron cross sections are similar for the two materials whilst the number of atoms in GaAs per unit mass is lower due to the larger atomic weight. On the other hand NIEL for fast protons and pions is larger in GaAs than in $\mathrm{Si}$ (see fig 2). This can be understood from the Lindhard factors for the two materials, which are presented in fig 11. This figure shows that the threshold recoil energy, below which NIEL becomes dominant is approximately 7 times higher in GaAs than in Si due to the lower velocities of the heavier ions in the former. As a result the average NIEL deposited by a fast recoil in GaAs is higher than that in Si.

From this it can be seen that the ratio of NIEL for fast charged hadrons to NIEL for $1 \mathrm{Mev}$ neutrons (and hence the ratio of radiation damage) will be much higher in GaAs than in Si.

\section{Implications for the use of GaAs Detectors at the LHC}

This is illustrated by the proposed use of GaAs in the forward wheels of the SemiConductor Tracker (SCT) by the ATLAS Collaboration [16]. The computed pion fluences at the position of the GaAs wheels for the SCT is $0.1210^{14}$ pions per $\mathrm{cm}^{2}$ per year in the SCT at full luminosity [13,14]. This is between a factor 3-10 more than the number of expected neutrons depending on whether there is a polythene moderator in the apparatus or not. The fluences of other charged hadrons (eg protons and kaons) is expected to be 
much smaller than that for pions.

The NIEL per pion in the SCT has been computed to be $3.4 \mathrm{keV} \mathrm{gm}^{-1} \mathrm{~cm}^{2}$ by averaging the NIEL stopping powers in GaAs in fig 2 over the spectrum of pions expected in the vicinity of the interaction point given in [8]. From this we deduce that the total NIEL deposited in the GaAs will be $0.4110^{14} \mathrm{keV} \mathrm{gm}^{-1}$ per year where 1 year means an integrated luminosity in ATLAS of $10^{41} \mathrm{~cm}^{-2}$.

A MIP gives a signal of 26.6 thousand electons in a GaAs detector of thickness 200 microns with 100 per cent CCE. Hence it can be seen from fig 6 that if we constructed the forward SCT wheels with detectors similar to the types tested by $[3,4,5]$ at the outset we could expect a signal of 22.3 thousand electrons. Assuming that the CCE deteriorates at the rate given by the smooth curve in fig 6 , after a period of 2 years of operation this signal would decrease to 11.7 thousand electrons. The decrease in CCE would then slow down and after 4 years of running we would expect a signal of 9.3 thousand electrons. Finally after 10 years of operation (integrated luminosity of $7.310^{41} \mathrm{~cm}^{-2}$ ) we would expect a MIP signal of 7.4 thousand electrons. An independent calculation of this [5] gives 4.0 thousand electrons after 10 years at the LHC. The difference represents the uncertainty in the calculation.

\section{Conclusion}

Irradiated GaAs detectors made from materials from a wide variety of sources show a good correlation between CCE and total NIEL. This indicates that the reduction in performance of GaAs charged particle detectors can be ascribed to NIEL. Such detectors have been shown to be quite radiation hard to neutrons but are rather more sensitive to damage by charged hadrons and this has been shown to be both qualitatively and quantitatively understandable. The correlation between CCE and NIEL has been used to estimate the rate of deterioration which would be expected if the forward wheels of the SCT in ATLAS at the LHC were constructed of GaAs detectors. The data indicate that after an initial rapid deterioration in performance the decrease in CCE slows down and after the full lifetime of the LHC we might expect detectors which give a signal in the range 4.0 - 7.4 thousand electrons to MIPS.

\section{Acknowledgements}

We thank the organisers for creating such an interesting and stimulating workshop. We are grateful to the Aachen, Freiburg and Glasgow groups for providing us with their data. 
It is a pleasure to acknowledge the interest and encouragement of the RD 8 collaboration and in particular the spokespersons Professors C del Papa and K M Smith.

\section{References}

1. E A Burke C J Dale A B Campbell G P Summers T Palmer and R Zuleeg; IEEE Transactions on Nuclear Science Vol NS-34 (1987) 1220.

2. RD8; S P Beaumont et al; NIM A322 (1992) 472-482

3. Glasgow data, R Bates; talk at 1996 IOP meeting (Imperial College, London) and also presented at this meeting.

4. Aachen data, W Xiao; talk presented at this meeting and $\mathrm{K}$ Luebelsmeyer et al Aachen preprint PITHA 96/13

5. Freiburg data, M Rogalla; talk presented at this meeting.

6. A Van Ginneken; Fermilab preprint FN-522 (1989)

7. J Lindhard et al; Mat. Fys. Medd. Dan. Vid Selsk 33 (1963) Number 14

8. M Huhtinen and P Aarnio; Preprint HU-SEFT R 1993-02 and NIM A335 (1993) 580-582

9. T F Luera et al; IEEE Trans. Nucl. Sci. NS-34 (6) (1987) 1557.

10. A M Ougouag et al; IEEE Trans. Nucl. Sci. NS-37 (6) (1990) 2219.

11. M Edwards and D R Perry; Radiation Hardness Testing Facility, Rutherford Lab. Report RAL-90-065

12. I Lazanu et al; Proceedings of Int Conf on Radiation Effects, Florence, March 1996 (to be published in NIM)

13. G.Gorfine and G N Taylor; ATLAS Note INDET-NO-030

14. T Bowcock, A Clark and M Edwards ATLAS Internal Note INDET-NO-069 (Oct 1994) 
15. A Chilingarov and T Sloan; Proceedings of Int Conf on Radiation Effects, Florence, March 1996 (to be published in NIM)

16. ATLAS Technical Proposal (1994) CERN/LHCC/94-43.

17. V Chmill, presented at this meeting.

18. A Chilingarov, H Feick, E Fretwurst, G Lindstrom, S Roe and T Schulz Nuc. Inst. and Methods A360 (1995) 432. 


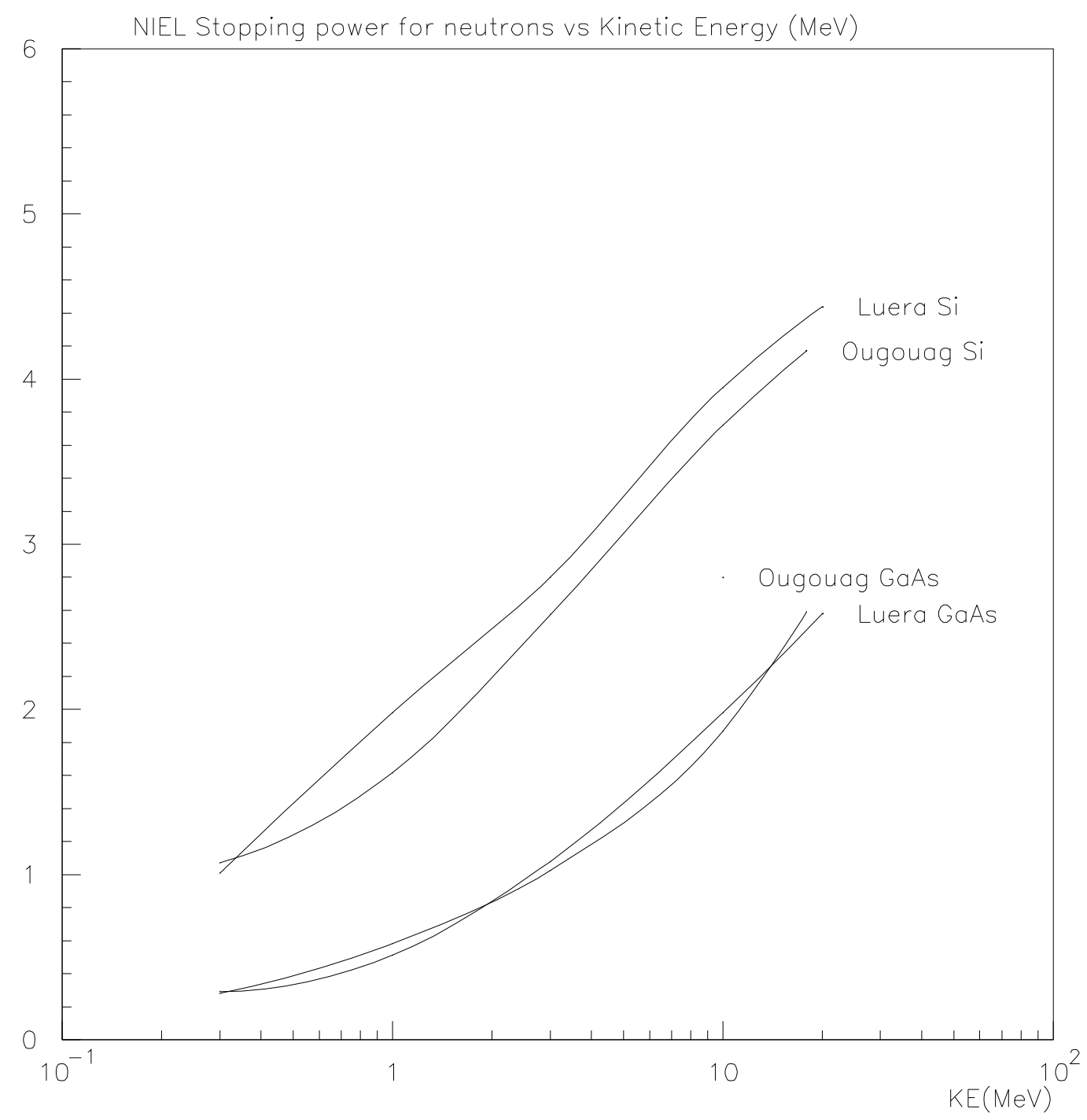

Fig 1. Calculations $[9,10]$ of the NIEL stopping power for neutrons in $\left(\mathrm{keV}\left(\mathrm{gm} \mathrm{cm}^{-2}\right)^{-1}\right)$ in $\mathrm{Si}$ and $\mathrm{GaAs}$ as a function of neutron kinetic energy (smoothed through nuclear resonances) 


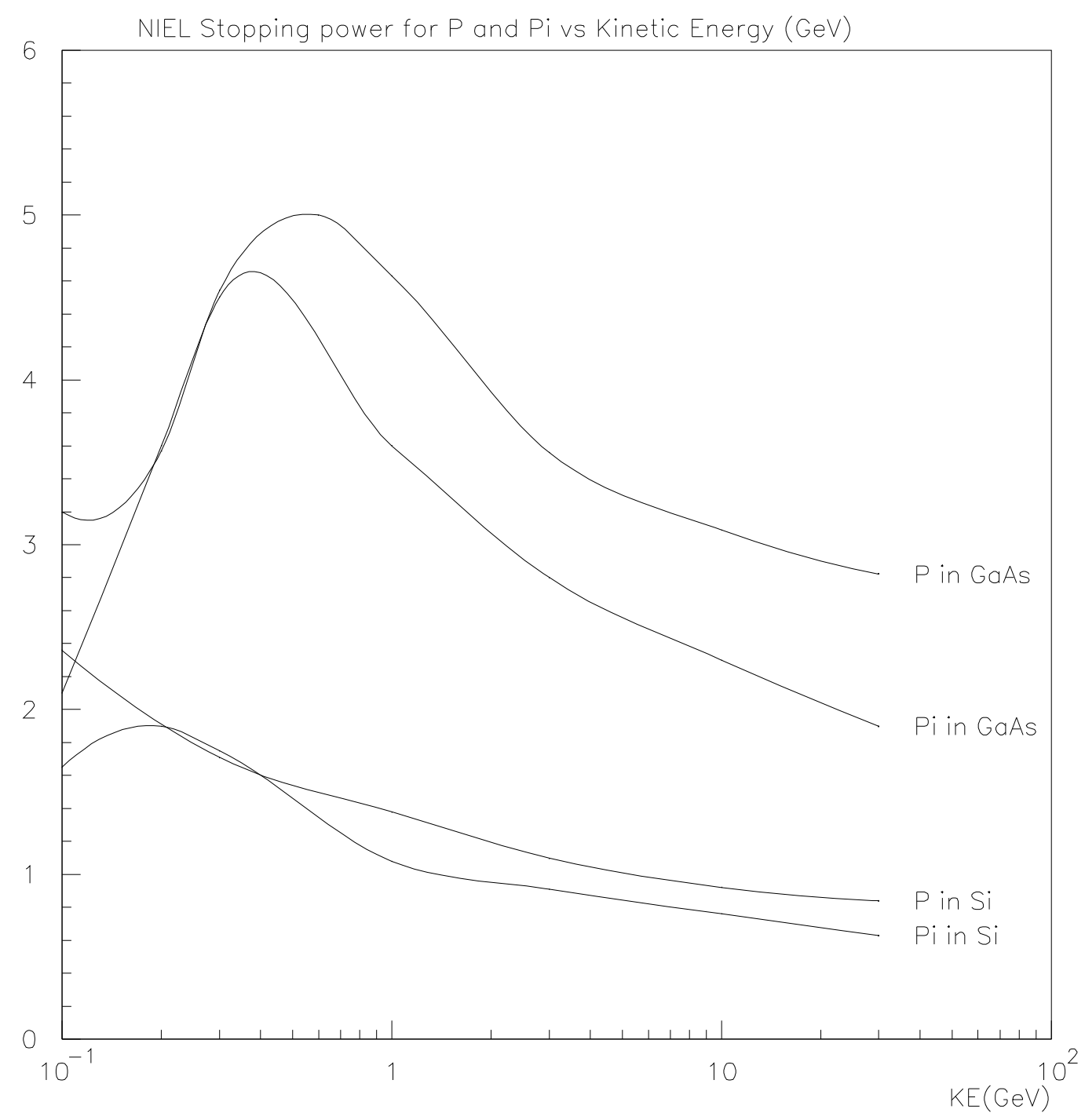

Fig 2. Calculations of the NIEL stopping power for protons and pions in $\left(\mathrm{keV}\left(\mathrm{gm} \mathrm{cm}^{-2}\right)^{-1}\right)$ in $\mathrm{Si}$ and $\mathrm{GaAs}$ as a function of kinetic energy. 


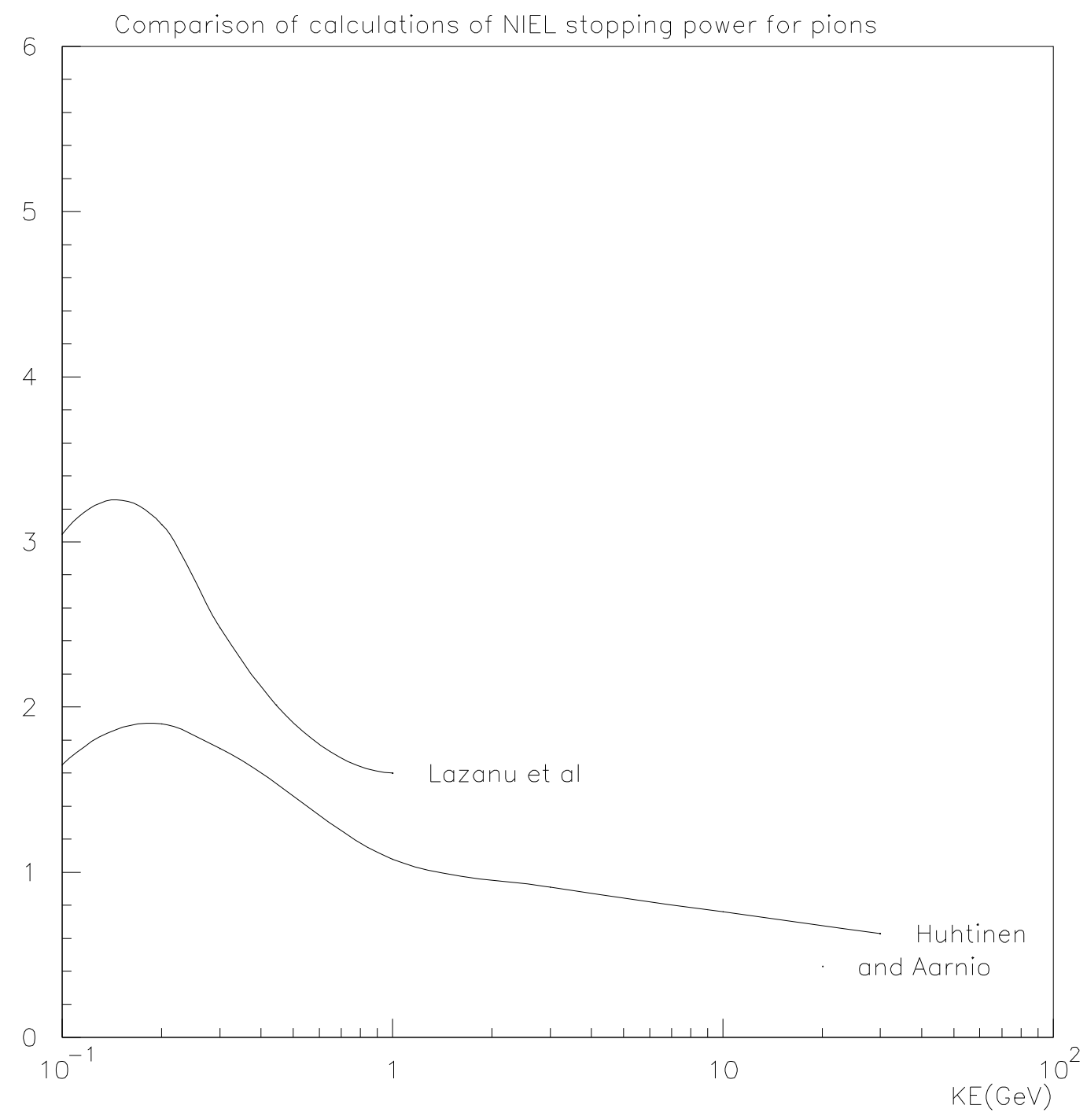

Fig 3 Comparison of NIEL stopping power calculations for pions in $\mathrm{Si}$ in $\left(\mathrm{keV}\left(\mathrm{gm} \mathrm{cm}^{-2}\right)^{-1}\right)$ from references [8] and [12]. 


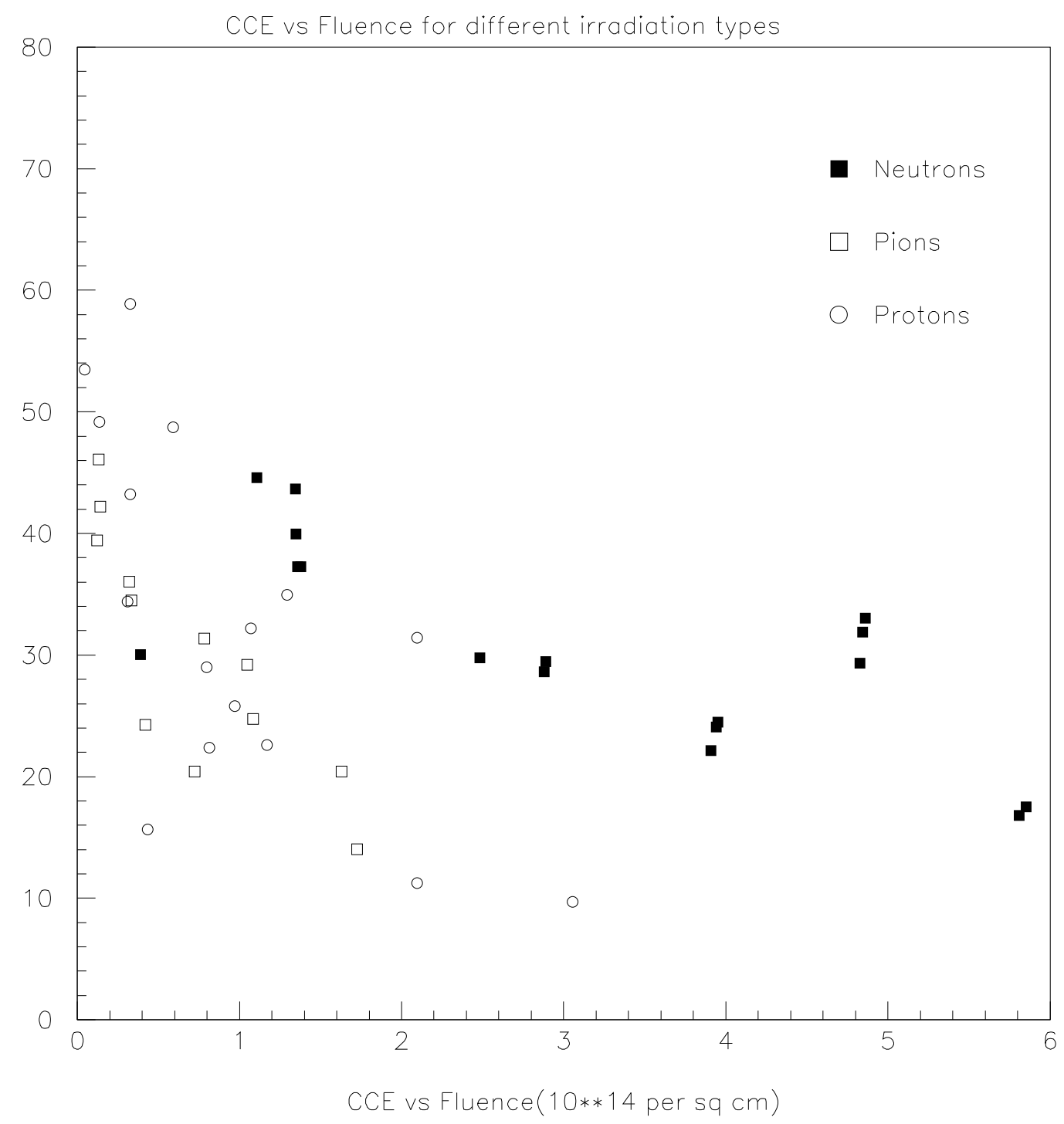

Fig $4 \mathrm{CCE}(\%)$ as a function of particle fluence (particles $\mathrm{cm}^{-2}$ ) for ISIS neutrons, 300 $\mathrm{MeV} / \mathrm{c}$ pions and $24 \mathrm{GeV} / \mathrm{c}$ protons. The data are from [3]. 


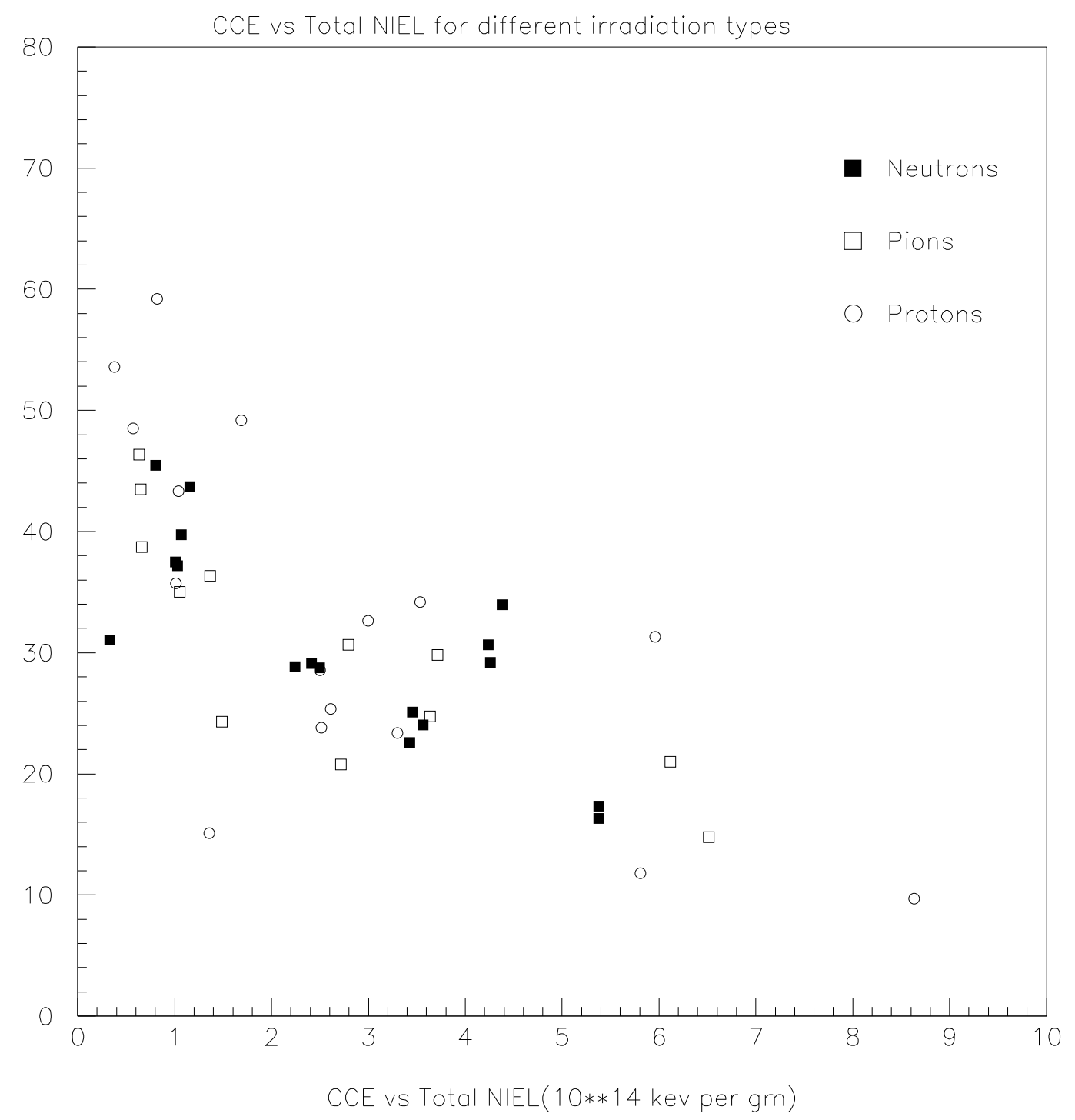

Fig $5 \mathrm{CCE}(\%)$ as a function of total NIEL for ISIS neutrons, $300 \mathrm{MeV} / \mathrm{c}$ pions and 24 $\mathrm{GeV} / \mathrm{c}$ protons. The data are from [3]. 


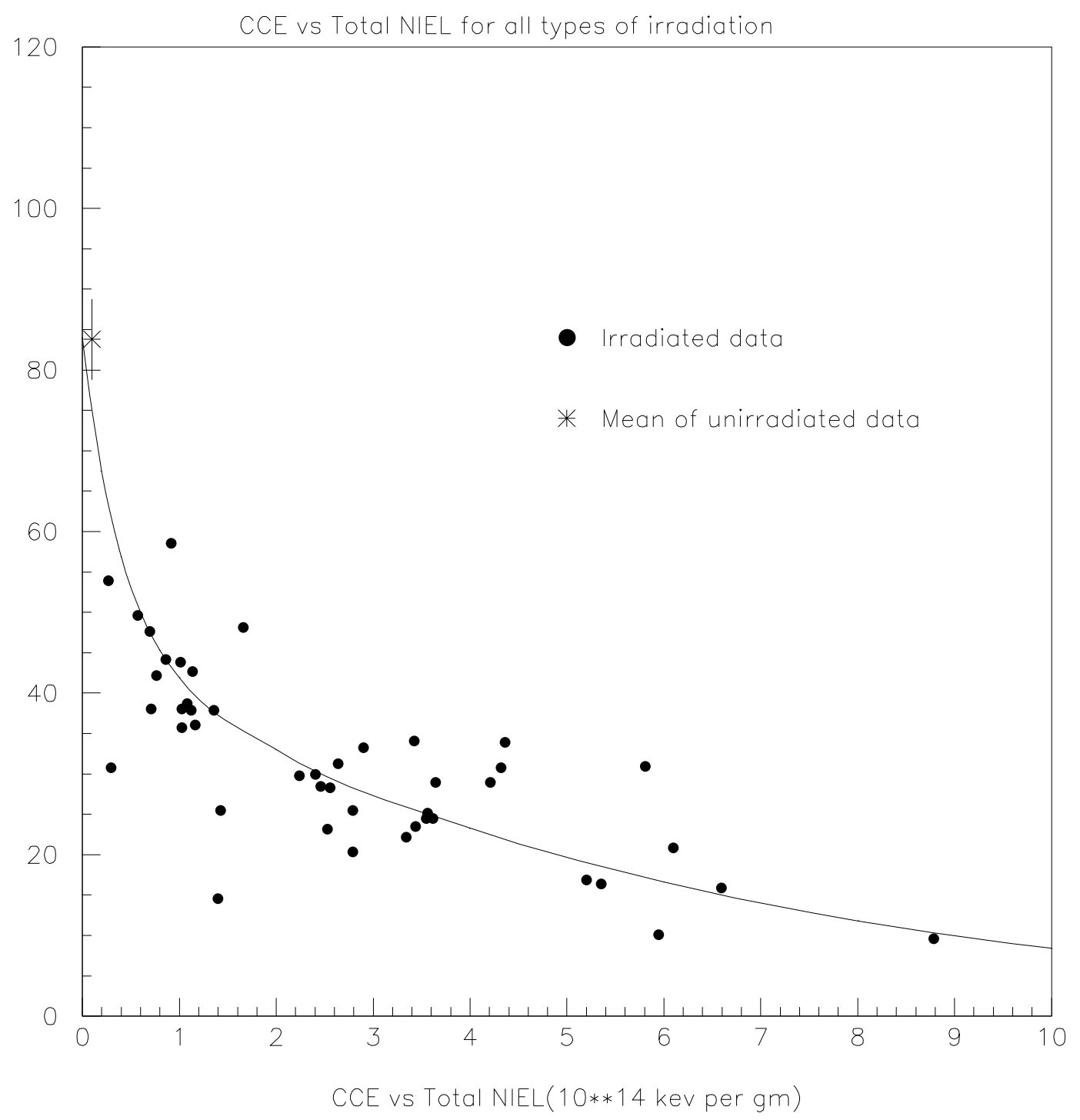

Fig 6 Measured CCE(\%) as a function of total NIEL from [3]. The smooth curve is the fit described in the text. 


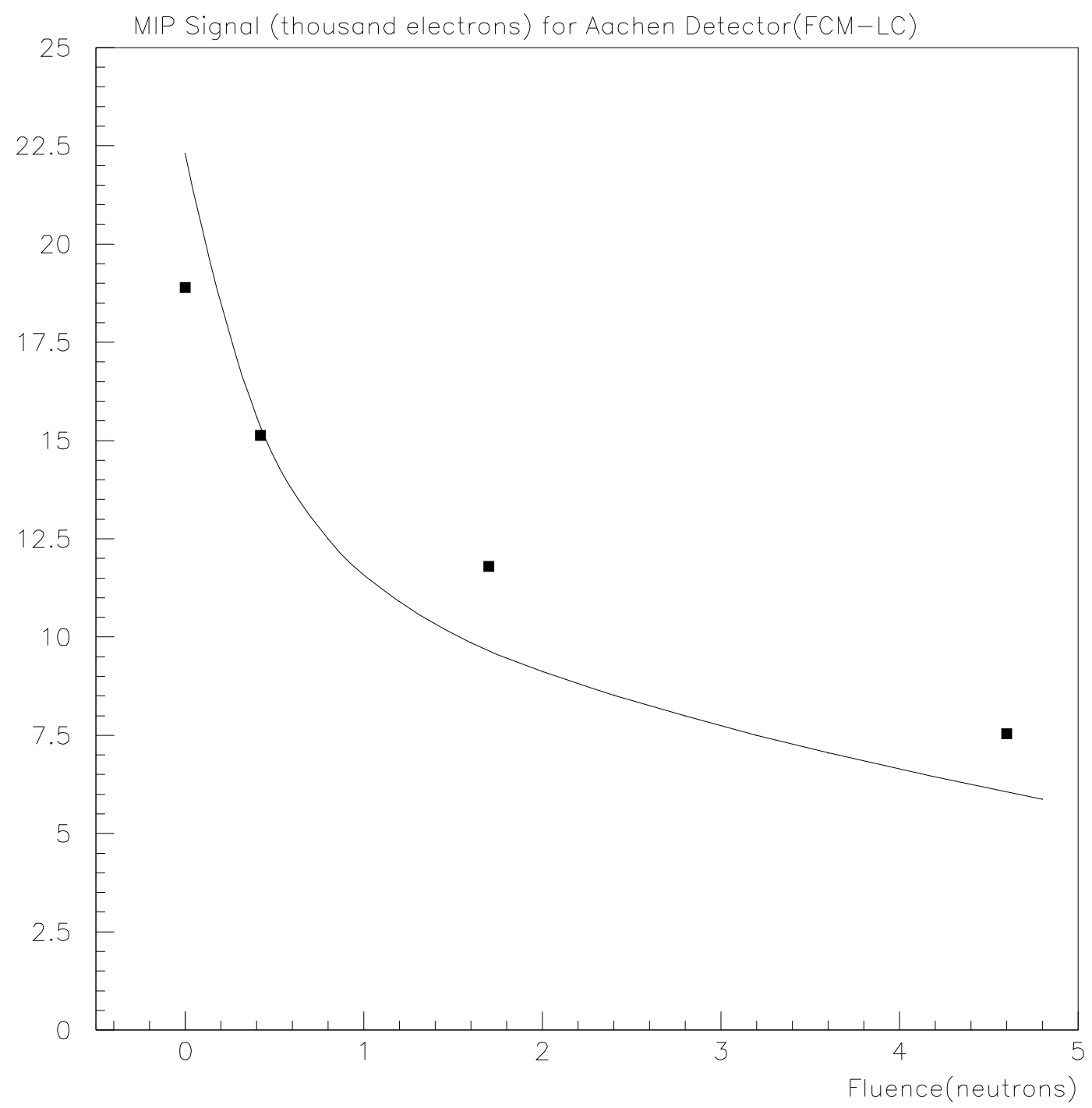

Fig 7 Measured MIP signal as a function of neutron fluence (in units of $10^{14} \mathrm{~cm}^{-2}$ ) from [4]. The smooth curve is from the fit to the Glasgow data [3] in fig.6. 

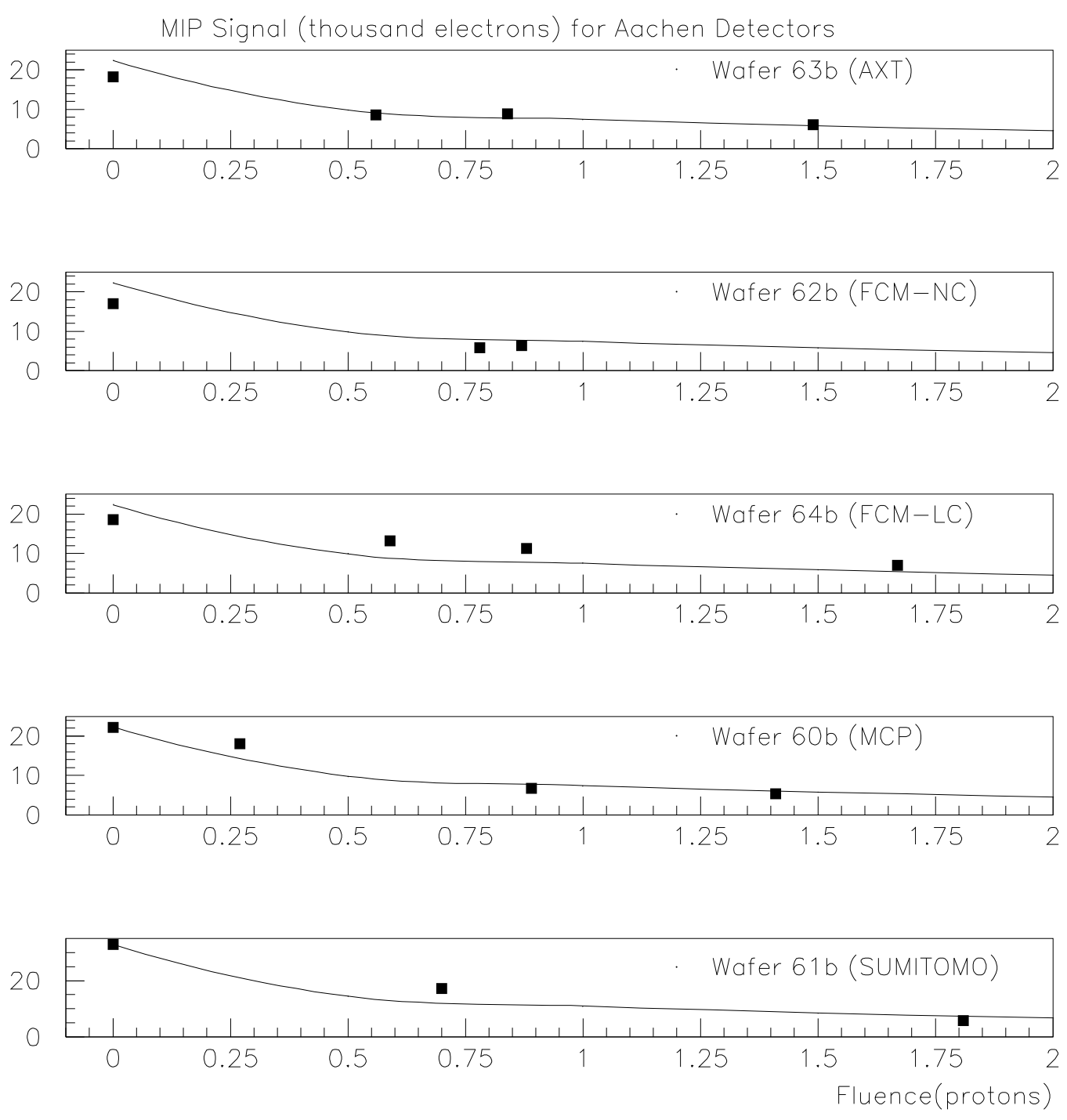

Fig 8 Measured MIP signal as a function of proton fluence (in units of $10^{14} \mathrm{~cm}^{-2}$ ) for different detectors from [4]. The smooth curve is from the fit to the Glasgow data[3] in fig.6. 

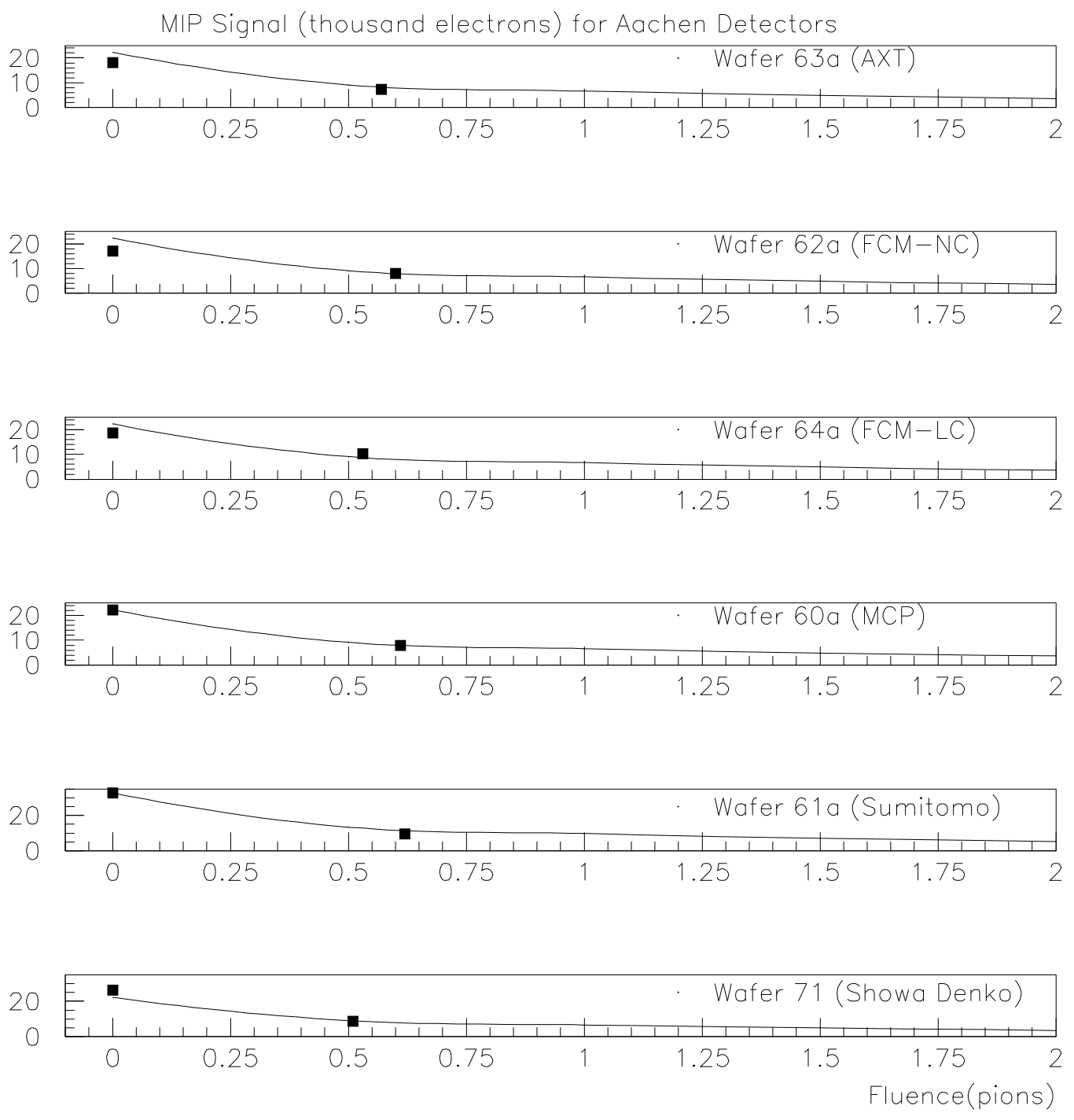

Fig 9 Measured MIP signal as a function of pion fluence (in units of $10^{14} \mathrm{~cm}^{-2}$ ) for different detectors from [4]. The smooth curve is from the fit to the Glasgow data[3] in fig.6. 


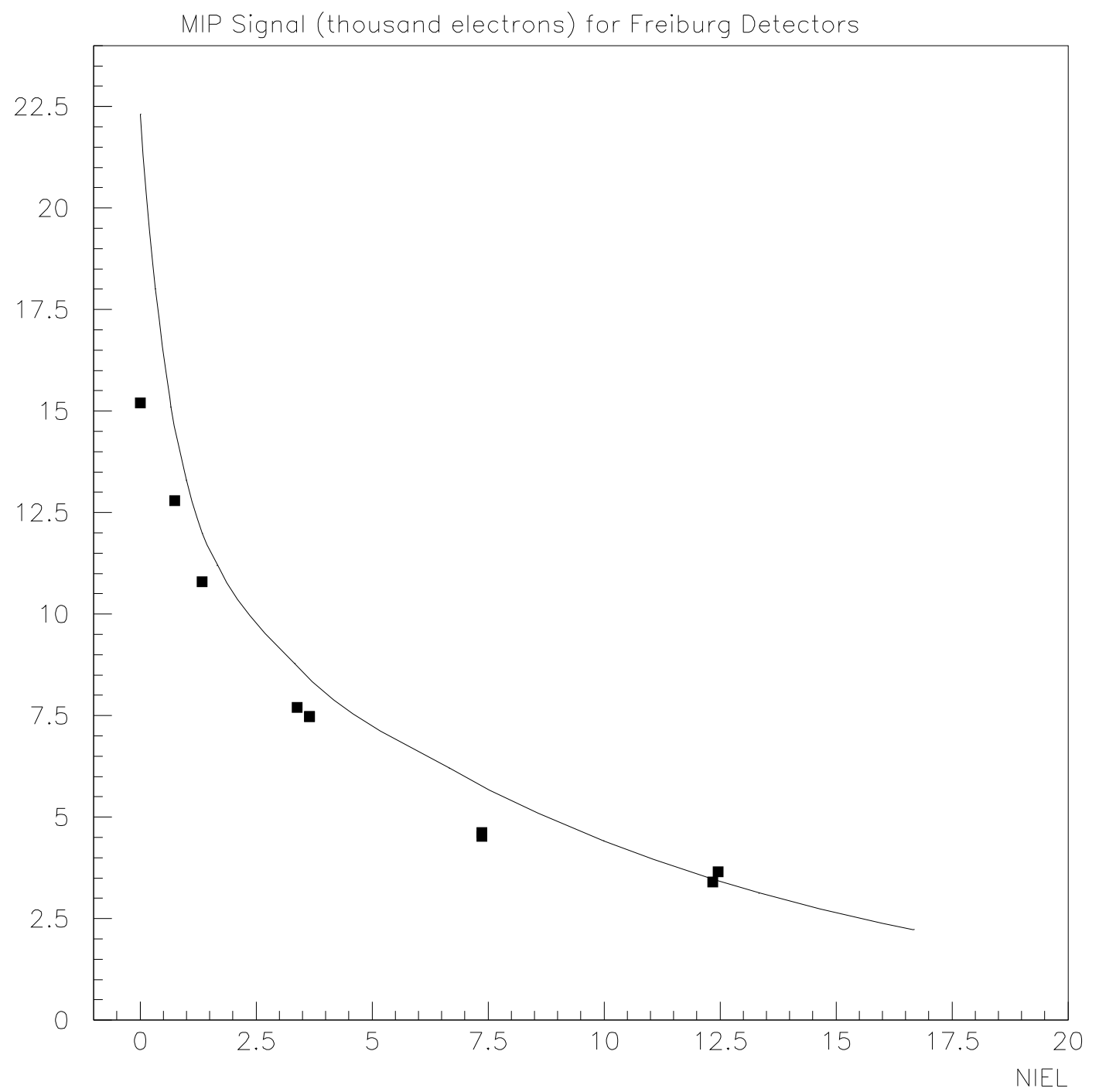

Fig 10 Measured MIP signal of samples from [5] irradiated by neutrons and protons as a function of $1 \mathrm{MeV}$ equivalent neutron fluence in units of $10^{14}$ neutrons per $\mathrm{cm}^{-2}$. The smooth curve is from the fit to the Glasgow data [3] in fig.6. 


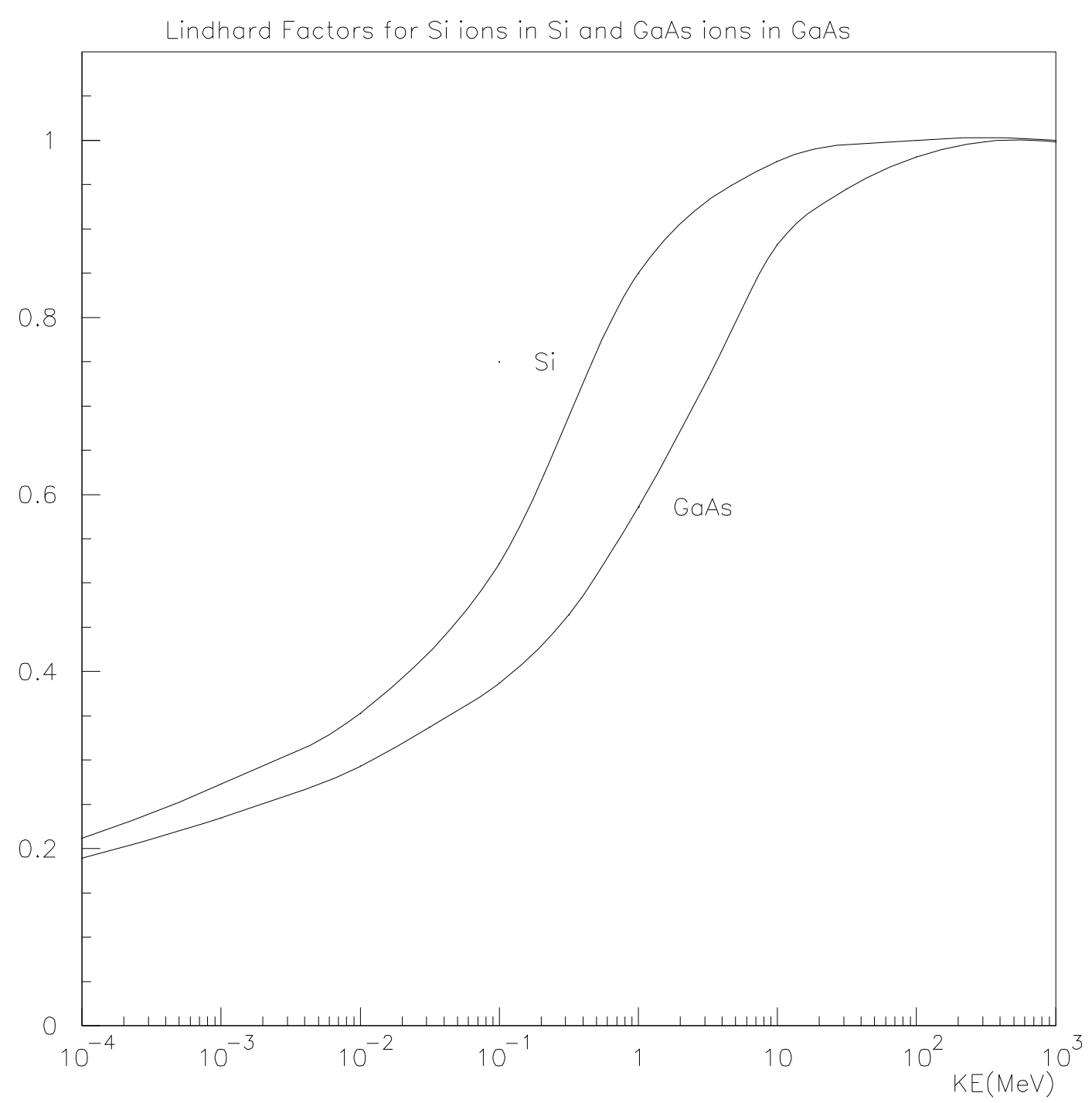

Fig 11 The total fraction of the energy appearing as ionization energy due to interactions with atomic electrons for GaAs ions in $\mathrm{GaAs}$ and $\mathrm{Si}$ ions in Si calculated according to the formulae of Lindhard et al [7]. 